\title{
OPTIMAL CONSUMPTION/PORTFOLIO CHOICE WITH BORROWING RATE HIGHER THAN DEPOSIT RATE
}

\author{
WENSHENG XU ${ }^{1}$ and SHUPING CHEN ${ }^{2}$
}

(Received 18 June 1996; revised 4 June 1997)

\begin{abstract}
In this paper, optimal consumption and investment decisions are studied for an investor who has available a bank account and a stock whose price is a log normal diffusion. The bank pays at an interest rate $r(t)$ for any deposit, and vice takes at a larger rate $r^{\prime}(t)$ for any loan. Optimal strategies are obtained via Hamilton-Jacobi-Bellman (HJB) equation which is derived from dynamic programming principle. For the specific HARA case, we get the optimal consumption and optimal investment explicitly, which coincides with the classical one under the condition $r^{\prime}(t) \equiv r(t)$.
\end{abstract}

\section{Introduction}

The central problem in any theory of economic growth is how resources should be split between investment and consumption. Investment is not an end in itself but is undertaken in order to provide more consumption later. Thus the theory is essentially dynamic. The investment model to be described in the following is due to R. C. Merton [3].

It is supposed that the investor has the choice of only two different investments. One of the assets is called bond (bank account) whose price $p_{0}(t)$ is assumed to satisfy an ordinary differential equation:

$$
d p_{0}(t)= \begin{cases}r(t) p_{0}(t) d t, & p_{0}(t) \geq 0 \\ r^{\prime}(t) p_{0}(t) d t, & p_{0}(t)<0\end{cases}
$$

where $r(t)$ is the interest rate and $r^{\prime}(t)$ is the borrowing rate, which is larger that $r(t)$ in general. The other asset is risky, called stock, whose price is assumed to admit a

\footnotetext{
'Department of Applied Mathematics, Zhejiang University, Hangzhou, 310027, P. R. China.

${ }^{2}$ Department of Applied Mathematics, Zhejiang University, Hangzhou, 310027, P. R. China.

(C) Australian Mathematical Society, 1998, Serial-fee code 0334-2700/98
} 
$\log$ normal diffusion

$$
d p(t)=b(t) p(t) d t+\sigma(t) p(t) d W_{t},
$$

where $b(t)$ represents the instantaneous expected rate of return, $\sigma(t)$ the instantaneous volatility. Here $W$, is a one-dimensional Brownian motion on some given probability space $\left(\Omega, \mathscr{F}, P, \mathscr{F}_{t}\right), \mathscr{F}_{t}$ is the natural filtration, that is,

$$
\mathscr{F}_{1}=\sigma\left\{W_{s}: 0 \leq s \leq t\right\} .
$$

Here and below we make the following assumption.

(H1) All the coefficients $r(t), r^{\prime}(t), b(t)$ and $\sigma(t)$ are assumed to be deterministic and bounded uniformly in $t \in[0, T]$. Furthermore, it is natural to assume that $r(t) \leq r^{\prime}(t) \leq b(t)$

We denote by $\pi(t)$ the amount invested in the stock, $c(t)$ the consumption rate process and $w(t)$ the wealth of the investor. We assume that $\left(c_{t}, \pi_{t}\right) \in L_{\mathscr{F}}^{2}(0, T) \times$ $L_{\mathscr{F}}^{2}(0, T)$, where $L_{\mathscr{F}}^{2}(0, T)$ is the space of all $\mathscr{F}_{t}$-adapted processes $v(t)$ such that

$$
E \int_{0}^{T} v^{2}(t) d t<\infty
$$

We allow $\pi(t)$ to become negative, which represents selling the stock short. Similarly the amount of money $w(t)-\pi(t)$ invested in the bond (bank account) may also be negative, and this is to be interpreted as borrowing from the bank at rate $r^{\prime}(t)$. It is assumed that both of these are allowed, but cannot be unbounded. We have $c(t) \geq 0$ for all $t \in[0, T]$ since consumption is essentially non-negative.

At each moment $t$, the investor may choose freely his consumption $c(t)$ and the number of dollars $\pi(t)$ invested in the risky asset. We denote by $\mathscr{U}_{a d}$ the set of these admissible pairs $(c ., \pi$.). The wealth of investor who starts at initial time $s$ with some initial endowment $w$ may be modelled by

$$
\begin{aligned}
d w(t)= & {[r(t) w(t)-c(t)+(b(t)-r(t)) \pi(t)} \\
& \left.-\left(r^{\prime}(t)-r(t)\right)(w(t)-\pi(t))^{-}\right] d t+\sigma(t) \pi(t) d W_{t}, \\
w(s)= & w .
\end{aligned}
$$

Suppose the investor wants to maximise the expected utility

$$
J(c ., \pi . ; s, w)=E\left\{\int_{s}^{T} U(t, c(t)) d t+B(T, w(T))\right\}
$$

by making appropriate consumption/portfolio decisions $\left(c ., \pi\right.$.) $\in \mathscr{U}_{a d}$. Here $\left(\bar{c} ., \bar{\pi}_{.}\right)$ is called an optimal strategy if it attains the maximum of $J(c ., \pi ; s, w)$.

The constraint is 
(H2) $w(t) \geq 0$ for all $t \in[0, T]$ (the investor must be solvent).

Obviously maximisation of $J(c ., \pi . ; s, w)$ over $\left(c ., \pi\right.$.) $\in \mathscr{U}_{a d}$ now constitutes a completely specified stochastic optimal control problem. For $r^{\prime}(t) \equiv r(t)$, these results are due to R. C. Merton [3]. For the specific HARA case, see [2] for reference, that is,

$$
\begin{aligned}
U(t, c) & =L e^{-\beta t} \frac{c^{1-R}}{1-R}, \\
B(T, w) & =K \frac{w^{1-R}}{1-R},
\end{aligned}
$$

with $R \in(0,1), \beta>0, L>0$ and $K>0$, it turns out that this stochastic optimal control problem is one of the few nonlinear stochastic control problems which can be explicitly solved. We have the following result.

THEOREM 1. Suppose $r^{\prime}(t) \equiv r(t)$. For the specific HARA case, the optimal investment and optimal consumption rules to the optimal decision problem (3) and (4) are given by

$$
\pi^{*}(s)=\frac{b(s)-r(s)}{R \sigma^{2}(s)} w(s)
$$

and

$$
c^{*}=\bar{C}(s) w(s)
$$

respectively, with $\bar{C}(s)$ a given deterministic function. Furthermore, the value function is $v(s, w)=v(s) w^{1-R}(s)$ with $v(s)$ some given deterministic function, where $v(s, w)$ is defined by

$$
v(s, w):=\max _{(c ., \pi .) \in \mathscr{U}_{a d}} J(c ., \pi ; s, w)
$$

This result is an application of the dynamic programming method. The corresponding $\mathrm{HJB}$ equation fortunately can be explicitly solved under this circumstance.

In the real world, the borrowing rate $r^{\prime}(t)$ is always larger than the interest rate $r(t)$ in the same region and at the same time. Therefore, generally speaking, the assumption $r^{\prime}(t) \equiv r(t)$ made in Theorem 1 is improper in some sense. In this paper, we consider the optimal choice problem (3) and (4) for the general case $r^{\prime}(t) \geq r(t)$. The difficulty is that the state equation (3) is nonlinear. Also following the dynamic programming principle, we get the corresponding HJB equation. Thus the optimal decisions can be derived in an abstract form. For the specific HARA case, we get the optimal investment and optimal consumption in closed form analogous to that of Theorem 1. This result is interesting, and is new to the authors' knowledge. 
This paper is organised as follows. We state our problem in detail in Section 2, and obtain the optimal solutions in an abstract form via the dynamic programming method. Section 3 is devoted to the specific HARA case, where optimal choices in closed form are obtained and we give a conclusion in Section 4 .

\section{Statement of our problem and optimal decisions}

In this section, we first restate our optimal control problem (3) and (4) in detail, and then solve it via the dynamic programming method.

Let $\left(\Omega, \mathscr{F}, P, \mathscr{F}_{t}\right)$ be a given probability space, and $W_{t}$ a one-dimensional Brownian motion on it. Here $\mathscr{F}_{t}$ is the natural filtration of $W_{l}$, that is,

$$
\mathscr{F}_{t}=\sigma\left\{W_{r}-W_{s}: s \leq r \leq t\right\}
$$

with $s$ the initial time.

Consider the stochastic control system (wealth equation):

$$
\begin{aligned}
d w(t)= & {[r(t) w(t)-c(t)+(b(t)-r(t)) \pi(t)} \\
& \left.-\left(r^{\prime}(t)-r(t)\right)(w(t)-\pi(t))^{-}\right] d t+\sigma(t) \pi(t) d W_{t}, \\
w(s)= & w,
\end{aligned}
$$

where $(c ., \pi.) \in \mathscr{U}_{a d}$ is regarded as control, and all the coefficients satisfy the assumption (H1) made in Section 1.

Our intent is to maximise the expected utility

$$
J(c, \pi ; s, w)=E\left\{\int_{s}^{T} U(t, c(t)) d t+B(T, w(T))\right\}
$$

over the set $\mathscr{U}_{a d}$ of admissible controls with the constraint (H2).

We assume also

(H3) $U(t, c)$ and $B(T, w)$ are strictly increasing, convex and differentiable with respect to $c$ and $w$ respectively.

The above assumption means that the investor prefers higher levels of consumption and terminal wealth to lower levels, but that he is also risk averse, that is, that his marginal utilities $(\partial / \partial c) U(t, c)$ and $(\partial / \partial w) B(t, w)$ are decreasing in the arguments $c$ and $w$ respectively.

The celebrated dynamic programming principle is valid for solving this optimal control problem. For our purpose, we define the value function

$$
v(s, w):=\sup _{(c . \pi .) \in \mathscr{U}_{a d}} J(c, \pi . ; s, w) .
$$


Here and below, we define $v_{w}$ as the partial derivative of $v$ with respect to $w$ and $v_{w w}$ as the second-order partial derivative of $v$ with respect to $w$.

Because of (H3), it can be checked that $v_{w} \geq 0$ and $v_{w w}<0$, which means that the value function is a nondecreasing convex function. Applying the optimal principle and Ito's lemma easily yields the following HJB equation:

$$
\begin{aligned}
& v_{s}+r(s) w v_{w}+\sup _{c}\left\{U(s, c)-v_{w} c\right\} \\
& \quad+\sup _{\pi}\left\{(b(s)-r(s)) v_{w} \pi-\left(r^{\prime}(s)-r(s)\right)(w-\pi)^{-}+\frac{1}{2} \sigma^{2}(s) v_{w w} \pi^{2}\right\}=0, \\
& v(T, w)=B(T, w) .
\end{aligned}
$$

Suppose $\left(c^{*}, \pi_{.}^{*}\right)$ is optimal. By $(8)$, we have $U_{c}^{\prime}\left(s, c^{*}\right)=v_{w} \geq 0$. Since $U_{c}^{\prime}(s, c)$ is monotonically decreasing with respect to $c$, its inverse exists and is denoted by $g$. Thus the optimal consumption rule is given by

$$
c^{*}(s)=g\left(s, v_{w}\right),
$$

via $v$ and $g$, and

$$
\sup _{c}\left\{U(s, c)-v_{w} c\right\}=U\left(s, g\left(s, v_{w}\right)\right)-v_{w} g\left(s, v_{w}\right) .
$$

We denote

$$
\begin{aligned}
& f(\pi)=(b(s)-r(s)) v_{w} \pi-\left(r^{\prime}(s)-r(s)\right)(w-\pi)^{-}+\frac{1}{2} \sigma^{2}(s) v_{w w} \pi^{2}, \\
& f_{1}(\pi)=(b(s)-r(s)) v_{w} \pi+\frac{1}{2} \sigma^{2}(s) v_{w w} \pi^{2} \\
& f_{2}(\pi)=\left(b(s)-r^{\prime}(s)\right) v_{w} \pi+\frac{1}{2} \sigma^{2}(s) v_{w w} \pi^{2}+\left(r^{\prime}(s)-r(s)\right) w v_{w} .
\end{aligned}
$$

Obviously

$$
f(\pi)= \begin{cases}f_{1}(\pi), & w-\pi \geq 0 \\ f_{2}(\pi), & w-\pi>0\end{cases}
$$

For our purpose, we consider $\sup _{\pi} f_{1}(\pi)$ and $\sup _{\pi} f_{2}(\pi)$ respectively below.

Since

$$
f_{1}(\pi)=\frac{1}{2} \sigma^{2}(s) v_{w w}\left\{\pi+\frac{(b(s)-r(s)) v_{w}}{\sigma^{2}(s) v_{w w}}\right\}^{2}-\frac{1}{2} \frac{(b(s)-r(s))^{2} v_{w}^{2}}{\sigma^{2}(s) v_{w w}},
$$

we have

$$
\sup _{\pi} f_{1}(\pi)=-\frac{1}{2} \frac{(b(s)-r(s))^{2} v_{w}^{2}}{\sigma^{2}(s) v_{w w}}
$$


and this supremum is attained at

$$
\pi_{1}^{*}(s)=-\frac{(b(s)-r(s)) v_{w}}{\sigma^{2}(s) v_{w w}} .
$$

Similarly

$$
\begin{aligned}
f_{2}(\pi)= & \frac{1}{2} \sigma^{2}(s) v_{w w}\left\{\pi+\frac{\left(b(s)-r^{\prime}(s)\right) v_{w}}{\sigma^{2}(s) v_{w w}}\right\}^{2}-\frac{1}{2} \frac{\left(b(s)-r^{\prime}(s)\right)^{2} v_{w}^{2}}{\sigma^{2}(s) v_{w w}} \\
& +\left(r^{\prime}(s)-r(s)\right) w v_{w},
\end{aligned}
$$

so

$$
\sup _{\pi} f_{2}(\pi)=-\frac{1}{2} \frac{\left(b(s)-r^{\prime}(s)\right)^{2} v_{w}^{2}}{\sigma^{2}(s) v_{w w}}+\left(r^{\prime}(s)-r(s)\right) w v_{w},
$$

and this supremum is attained at

$$
\pi_{2}^{*}(s)=-\frac{\left(b(s)-r^{\prime}(s)\right) v_{w}}{\sigma^{2}(s) v_{w w}} .
$$

Now we proceed to consider $\sup _{\pi} f(\pi)$. Noticing $\pi_{2}^{*}(s) \leq \pi_{1}^{*}(s)$, we discuss it in the following three cases.

Case I. Suppose $w \leq \pi_{2}^{*}(s)$.

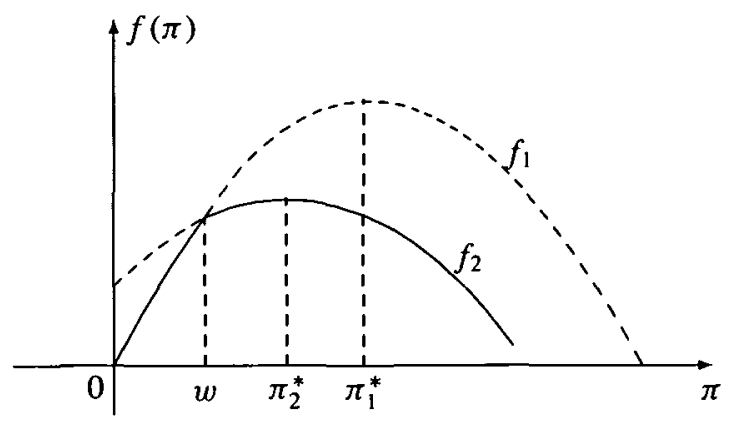

FIGURE 1.

See Figure 1 for illustration. Under these circumstances, we have

$$
\sup _{\pi} f(\pi)=\sup _{\pi} f_{2}(\pi)=-\frac{1}{2} \frac{\left(b(s)-r^{\prime}(s)\right)^{2} v_{w}^{2}}{\sigma^{2}(s) v_{w w}}+\left(r^{\prime}(s)-r(s)\right) w v_{w},
$$

and the supremum is attained at

$$
\pi^{*}(s)=\pi_{2}^{*}(s)=-\frac{\left(b(s)-r^{\prime}(s)\right) v_{w}}{\sigma^{2}(s) v_{w w}} .
$$


Then the corresponding HJB equation (8) can be written as

$$
\begin{aligned}
& v_{s}+r^{\prime}(s) w v_{w}+U\left(s, g\left(s, v_{w}\right)\right)-g\left(s, v_{w^{\prime}}\right) v_{w^{\prime}}-\frac{1}{2} \frac{\left(b(s)-r^{\prime}(s)\right)^{2} v_{w}^{2}}{\sigma^{2}(s) v_{w w}}=0, \\
& v(T, w)=B(T, w) .
\end{aligned}
$$

Therefore one can conclude that the optimal consumption and optimal investment are given by (9) and (17) respectively, provided that $w \leq \pi_{2}^{*}(s)$, where $v(s, w)$ is the solution of (18).

Case II. Suppose $\pi_{2}^{*}(s)<w<\pi_{1}^{*}(s)$.

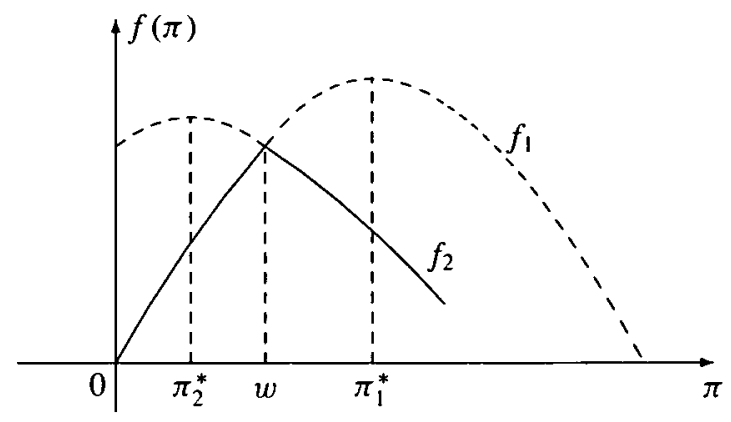

FIGURE 2.

See Figure 2 for illustration. Under these conditions, we have obviously

$$
\pi^{*}(s)=w(s)
$$

and

$$
\sup _{\pi} f(\pi)=f(w)=(b(s)-r(s)) w v_{w}+\frac{1}{2} \sigma^{2}(s) w^{2} v_{w w} .
$$

As a result, the corresponding HJB equation (8) has the form

$$
\begin{aligned}
& v_{s}+b(s) w v_{w}+U\left(s, g\left(s, v_{w}\right)\right)-g\left(s, v_{w}\right) v_{w}+\frac{1}{2} \sigma^{2}(s) w^{2} v_{w w}=0, \\
& v(T, w)=B(T, w) .
\end{aligned}
$$

Thus one can conclude that the optimal decisions are given by (9) and (19), provided that $\pi_{2}^{*}(s)<w<\pi_{1}^{*}(s)$, where $v(s, w)$ is the solution of $(21)$.

Case III. Suppose $w \geq \pi_{1}^{*}(s)$.

See Figure 3 for illustration. In this situation, we have

$$
\sup _{\pi} f(\pi)=\sup _{\pi} f_{1}(\pi)=-\frac{1}{2} \frac{(b(s)-r(s))^{2} v_{w}^{2}}{\sigma^{2}(s) v_{w w}},
$$


and the supremum is attained at

$$
\pi^{*}(s)=\pi_{1}^{*}(s)=-\frac{(b(s)-r(s)) v_{w}}{\sigma^{2}(s) v_{w w}}
$$

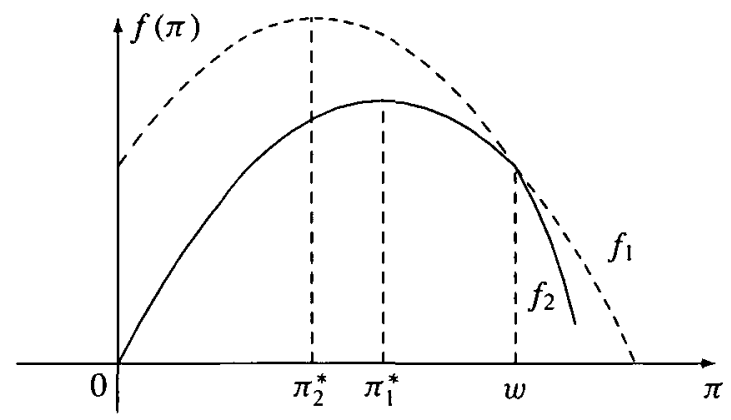

FIGURE 3.

Consequently, the HJB equation (8) can be rewritten as

$$
\begin{aligned}
& v_{s}+r(s) w v_{w}+U\left(s, g\left(s, v_{w}\right)\right)-g\left(s, v_{w}\right) v_{w}-\frac{1}{2} \frac{(b(s)-r(s))^{2} v_{w}^{2}}{\sigma^{2}(s) w^{2} v_{w w}}=0 \\
& v(T, w)=B(T, w)
\end{aligned}
$$

Thus one can conclude that the optimal choices are given by (9) and (23) respectively, provided that $w \geq \pi_{1}^{*}(s)$, where $v(s, w)$ is the solution of (24).

Generally speaking, the evaluation of the partial differential equations (18), (21) and (24) can be performed by appropriate algorithms. These algorithms can be developed using the ideas reported in [1] and [4]. The details are the subject of another investigation.

Thus the optimal choices can be calculated according to the above conclusions. Nevertheless, for the specific HARA case, we can solve it explicitly as below.

\section{The specific HARA case}

In this section, we consider the specific HARA case which can be solved explicitly, that is,

$$
\begin{aligned}
U(t, c) & =L e^{-\beta t} \frac{c^{1-R}}{1-R} \\
B(T, w) & =K \frac{w^{1-R}}{1-R}
\end{aligned}
$$


with $R \in(0,1)$ and $\beta>0$ to be the discount factor.

It is easily calculated that

$$
c^{*}(s)=g\left(s, v_{w}\right)=\left(\frac{e^{\beta_{s}}}{L} v_{w}\right)^{-1 / R}
$$

and

$$
\sup \left\{U(s, c)-v_{w} c\right\}=\frac{R}{1-R}\left(\frac{e^{\beta_{s}}}{L}\right)^{-1 / R} v_{w}^{1-1 / R}
$$

Case I. Suppose $w \leq \pi_{2}^{*}(s)$.

Then the corresponding HJB equation (18) takes the form

$$
\begin{aligned}
& v_{s}+r^{\prime}(s) w v_{w}+\frac{R}{1-R}\left(\frac{e^{\beta_{s}}}{L}\right)^{-1 / R} v_{w}^{1-1 / R}-\frac{1}{2} \frac{\left(b(s)-r^{\prime}(s)\right)^{2} v_{w}^{2}}{\sigma^{2}(s) v_{w w}}=0, \\
& v(T, w)=K \frac{w^{1-R}}{1-R} .
\end{aligned}
$$

Now we try to solve the above equation. Letting $v(s, w)=\phi(s) \frac{w^{1-R}}{1-R}$, it follows from (27) that

$$
\begin{aligned}
& \dot{\phi}+(1-R)\left[r^{\prime}(s)+\frac{\left(b(s)-r^{\prime}(s)\right)^{2}}{2 R \sigma^{2}(s)}\right] \phi+R\left(\frac{e^{\beta_{s}}}{L}\right)^{-1 / R} \phi^{1-1 / R}=0, \\
& \phi(T)=K .
\end{aligned}
$$

Obviously (28) is a Bernoulli-type equation. Denoting

$$
\begin{aligned}
& g_{1}(s)=(1-R)\left[r^{\prime}(s)+\frac{\left(b(s)-r^{\prime}(s)\right)^{2}}{2 R \sigma^{2}(s)}\right], \\
& g_{2}(s)=R\left(\frac{e^{\beta_{s}}}{L}\right)^{-1 / R},
\end{aligned}
$$

then (29) is rewritten as

$$
\begin{aligned}
& \dot{\phi}+g_{1}(s) \phi+g_{2}(s) \phi^{1-1 / R}=0, \\
& \phi(T)=K .
\end{aligned}
$$

Letting $\tilde{\phi}(s)=\phi(s) \exp \left(-\frac{1}{R} \int_{s}^{T} g_{1}(r) d r\right)$, it follows that

$$
\begin{aligned}
& \dot{\tilde{\phi}}+g_{2}(s) \exp \left(-\frac{1}{R} \int_{s}^{T} g_{1}(r) d r\right) \phi^{1-1 / R}=0, \\
& \tilde{\phi}(T)=K
\end{aligned}
$$


It is easily seen that

$$
\tilde{\phi}(s)^{1 / R}=K^{1 / R}+\frac{1}{R} \int_{s}^{T} g_{2}(t) \exp \left(-\frac{1}{R} \int_{s}^{T} g_{1}(r) d r\right) d t,
$$

from which we have

$$
\phi(s)=\exp \left(\int_{s}^{T} g_{1}(r) d r\right)\left[K^{1 / R}+\frac{1}{R} \int_{s}^{T} g_{2}(t) \exp \left(-\frac{1}{R} \int_{s}^{T} g_{1}(r) d r\right) d t\right]_{(30)}^{R}
$$

Thus

$$
v(s, w)=\phi(s) \frac{w^{1-R}}{1-R} .
$$

Moreover, the optimal consumption is

$$
c^{*}(s)=\left(\frac{e^{\beta_{r}}}{L}\right)^{-1 / R} \phi(s)^{-1 / R} w(s)
$$

and the optimal portfolio is

$$
\pi^{*}(s)=\phi_{2}^{*}(s)=\frac{b(s)-r^{\prime}(s)}{R \sigma^{2}(s)} w(s)
$$

provided that $w \leq \pi_{2}^{*}(s)$, that is,

$$
\frac{b(s)-r^{\prime}(s)}{R \sigma^{2}(s)} \geq 1
$$

Therefore, one can conclude that if the borrowing rate $r^{\prime}(s)$ satisfies

$$
r^{\prime}(s) \leq b(s)-R \sigma^{2}(s),
$$

then the optimal consumption and optimal investment are given by (32) and (33) respectively. Furthermore, the value function is given by (31).

Case II. Suppose $\pi_{2}^{*}(s) \leq w<\pi_{1}^{*}(s)$.

The corresponding HJB equation (21) takes the form

$$
\begin{aligned}
& v_{s}+b(s) w v_{w}+\frac{R}{1-R}\left(\frac{e^{\beta s}}{L}\right)^{-1 / R} v_{w}^{1-(1 / R)}+\frac{1}{2} \sigma^{2}(s) w^{2} v_{w w}=0 \\
& v(T, w)=K \frac{w^{1-R}}{1-R}
\end{aligned}
$$


Letting $v(s, w)=\Phi(s) \frac{w^{1-R}}{1-R}$, it can be checked that

$$
\begin{aligned}
& \dot{\Phi}+(1-R)\left[b(s)-\frac{1}{2} R \sigma^{2}(s)\right] \Phi+R\left(\frac{e^{\beta s}}{L}\right)^{-1 / R} \Phi^{1-(1 / R)}=0, \\
& \Phi(T)=K
\end{aligned}
$$

Write

$$
g_{11}(s)=(1-R)\left[b(s)-\frac{1}{2} R \sigma^{2}(s)\right]
$$

Then

$$
\begin{aligned}
& \dot{\Phi}+g_{11}(s) \Phi+g_{2}(s) \Phi^{1-(1 / R)}=0 \\
& \Phi(T)=K
\end{aligned}
$$

This can be solved as

$$
\Phi(s)=\exp \left(\int_{s}^{T} g_{11}(r) d r\right)\left[K^{1 / R}+\frac{1}{R} \int_{s}^{T} g_{2}(t) \exp \left(-(1 / R) \int_{t}^{T} g_{11}(r) d r\right) d t\right]_{(35)}^{R} .
$$

Consequently

$$
v(s, w)=\Phi(s) \frac{w^{1-R}}{1-R},
$$

and from (13) and (14) we know that

$$
\pi_{1}^{*}(s)=\frac{b(s)-r(s)}{R \sigma^{2}(s)} w(s)
$$

and

$$
\pi_{2}^{*}(s)=\frac{b(s)-r^{\prime} s(s)}{R \sigma^{2}(s)} w(s) .
$$

Thus the condition $\pi_{2}^{*}(s)<w<\pi_{1}^{*}(s)$ is equivalent to

$$
r(s)<b(s)-R \sigma^{2}(s)<r^{\prime}(s) .
$$

Therefore one can conclude that, if condition (37) holds, then the optimal consumption and optimal investment are given by

$$
c^{*}(s)=\left(\frac{e^{\beta s}}{L}\right)^{-1 / R} \Phi(s)^{-1 / R} w(s)
$$

and (19) respectively. Furthermore, the value function is given by (36). 
Case III. Suppose $w>\pi_{1}^{*}(s)$.

The corresponding HJB equation (24) assumes the form

$$
\begin{aligned}
& v_{s}+r(s) w v_{w}+\frac{R}{1-R}\left(\frac{e^{\beta s}}{L}\right)^{-1 / R} v_{w}^{1-(1 / R)}-\frac{1}{2} \frac{(b(s)-r(s))^{2} v_{w}^{2}}{\sigma^{2}(s) v_{w w}}=0, \\
& v(T, w)=K \frac{w^{1-R}}{1-R} .
\end{aligned}
$$

As with (27), it is easily seen that

$$
v(s, w)=\bar{\phi}(s) \frac{w^{1-R}}{1-R}
$$

with

$$
\begin{gathered}
\bar{\phi}(s)=\exp \left(\int_{s}^{T} \bar{g}_{1}(r) d r\right)\left[K^{1 / R}+\frac{1}{R} \int_{s}^{T} g_{2}(t) \exp \left(-(1 / R) \int_{t}^{T} \bar{g}_{1}(r) d r\right) d t\right]^{R}, \\
\bar{g}_{1}(s)=(1-R)\left[r(s)+\frac{(b(s)-r(s))^{2}}{2 R \sigma(s)^{2}}\right]
\end{gathered}
$$

As a result, the optimal decisions are given by

$$
c^{*}(s)=\left(\frac{e^{\beta s}}{L}\right)^{-1 / R} \bar{\phi}(s)^{-1 / R} w(s)
$$

and

$$
\pi^{*}(s)=\frac{b(s)-r(s)}{R \sigma^{2}(s)} w(s)
$$

respectively.

In addition, the condition $w \geq \pi_{1}^{*}(s)$ is equivalent to

$$
r(s) \geq b(s)-R \sigma^{2}(s) .
$$

Therefore one can conclude that, if condition (44) holds, then the optimal decisions are given by (42) and (43) respectively. Furthermore, the value function is given by (40).

We conclude the above discussion with the following theorem.

THEOREM 2. Under all the assumptions made in Section 1 and Section 2, the optimal decisions to the optimal control problem (5) and (6) for the specific HARA case are given by

$$
\pi^{*}(s)= \begin{cases}\frac{b(s)-r^{\prime}(s)}{R \sigma^{2}(s)} w(s), & b(s)-R \sigma(s)^{2} \geq r(s) \\ w(s), & r(s)<b(s)-R \sigma(s)^{2}<r^{\prime}(s), \\ \frac{b(s)-r(s)}{R \sigma^{2}(s)} w(s), & r(s) \geq b(s)-R \sigma^{2}(s) .\end{cases}
$$


and

$$
c^{*}(s)= \begin{cases}\left(\frac{\mathrm{e}^{\beta s}}{L}\right)^{-1 / R} \phi(s)^{-1 / R} w(s), & b(s)-R \sigma(s)^{2} \geq r^{\prime}(s), \\ \left(\frac{e^{\beta . x}}{L}\right)^{-1 / R} \Phi(s)^{-1 / R} w(s), & r(s)<b(s)-R \sigma(s)^{2}<r^{\prime}(s) \\ \left(\frac{e^{\beta s}}{L}\right)^{-1 / R} \bar{\phi}(s)^{-1 / R} w(s), & r(s) \geq b(s)-R \sigma^{2}(s),\end{cases}
$$

respectively, where $\phi(s), \Phi(s)$ and $\bar{\phi}(s)$ are defined by (30), (35) and (41). Furthermore, the value function is given by

$$
v(s, w)= \begin{cases}\phi(s) \frac{w^{1-R}}{1-R}, & b(s)-R \sigma(s)^{2} \geq r^{\prime}(s), \\ \Phi(s) \frac{w^{1-R}}{1-R}, & r(s)<b(s)-R \sigma(s)^{2}<r^{\prime}(s), \\ \bar{\phi}(s) \frac{w^{1-R}}{1-R}, & r(s) \geq b(s)-R \sigma^{2}(s) .\end{cases}
$$

\section{Conclusion}

In this paper we obtain the optimal consumption and optimal investment to the optimal decision problem (5) and (6) in an abstract form (see (9), (17), (19) and (23)) via the HJB equation which is derived from the dynamic programming principle. For the specific HARA case, we obtain the optimal decisions in an explicit form (45) and (46). From Theorem 2 we see that $b(s)-R \sigma^{2}(s)$ is a very important economic index for our optimal strategies. For deeper insight, we define

$$
\gamma(s):=\max \left\{r(s), \min \left\{r^{\prime}(s), b(s)-R \sigma^{2}(s)\right\}\right\}
$$

Then the optimal portfolio $\pi^{*}(s)$ can be written

$$
\pi^{*}(s)=\frac{b(s)-\gamma(s)}{R \sigma^{2}(s)} w(s)
$$

which coincides with the classical result of Theorem 1 when $r^{\prime}(s) \equiv r(s)$. Furthermore, let

$$
\bar{g}(s)=(1-R)\left[\gamma(s)+\frac{b(s)-\gamma(s)}{2 R \sigma(s)^{2}}\right]
$$

and

$$
\varphi(s)=\exp \left(\int_{s}^{T} \bar{g}(r) d r\right)\left[K^{1 / R}+\frac{1}{R} \int_{s}^{T} g_{2}(t) \exp \left(-\frac{1}{R} \int_{t}^{T} \bar{g}(r) d r\right) d t\right]^{R}
$$


It follows that

$$
c(s)^{*}=\left(\frac{e^{\beta s}}{L}\right)^{-1 / R} \varphi(s)^{-1 / R} w(s)
$$

and

$$
v(s, w)=\varphi(s) \frac{w^{1-R}}{1-R}
$$

THEOREM 2'. Under all the assumptions made in Section 1 and Section 2, the optimal decisions to the optimal control problem (5) and (6) for the specific HARA case are given by (49) and (52). Moreover, the value function is given by (53).

Theorem $2^{\prime}$ shows that the investor should borrow $\$\left(\left(b(s)-r^{\prime}(s)\right) w(s) / R \sigma^{2}(s)-\right.$ $w(s)$ ) from the bank when the borrowing rate $r^{\prime}(s)$ is relatively lower $\left(r^{\prime}(s) \leq\right.$ $\left.b(s)-R \sigma^{2}(s)\right)$ to gain his best yield. When the interest rate $r(s)$ is relatively lower and the borrowing rate $r^{\prime}(s)$ is relatively higher $\left(r(s)<b(s)-R \sigma^{2}(s)<\right.$ $r^{\prime}(s)$ ), the investor should invest all his/her present wealth $\$ w(s)$ in the stock, neither borrow money from the bank nor deposit money in the bank. When the interest rate $r(s)$ becomes relatively higher $\left(r(s) \geq b(s)-R \sigma^{2}(s)\right)$, the investor should have $\$\left(w(s)-\left(b(s)-r^{\prime}(s) w(s) / R \sigma^{2}(s)\right)\right.$ savings in the bank.

\section{Acknowledgements}

The author thanks deeply the associate editor and the anonymous referees for suggesting valuable improvements to the presentation of the paper. This work was supported by the National Natural Science Foundation of China.

\section{References}

[1] H. T. Banks, J. M. Crowley and K. Kunisch, "Cubic spline approximation techniques for parameter estimation in distributed systems", IEEE Trans. Aüto. Control AC-28 (1983) 773-786.

[2] D. Duffie, Security Markets: Stochastic Models (Academic Press, Boston, 1988).

[3] R. C. Merton, "Optimal consumption and portfolio rules in a continuous-time model", J. Econ. Theory 3 (1971) 373-413.

[4] K. L. Teo and Z. S. Wu, Copmputational Methods for Optimizing Distributed Systems (Academic Press, 1994). 